\title{
A Sensitive Search for Predicted Methanol Maser Transitions with the Australia Telescope Compact Array
}

\author{
A. Chipman ${ }^{1}$, S. P. Ellingsen ${ }^{1,4}$, A. M. Sobolev ${ }^{2}$ and D. M. Cragg ${ }^{3}$ \\ ${ }^{1}$ School of Physical Sciences, Private Bag 37, University of Tasmania, Hobart 7001, Australia \\ ${ }^{2}$ Ural Federal University, Lenin Avenue 51, 620000 Ekaterinburg, Russia \\ ${ }^{3}$ School of Chemistry, 19 Rainforest Walk, Clayton Campus, Monash University, Victoria 3800, Australia \\ ${ }^{4}$ Email: Simon.Ellingsen@utas.edu.au
}

(RECEIVED September 9, 2016; ACCEPTED October 10, 2016)

\begin{abstract}
We have used the Australia Telescope Compact Array to search for a number of centimetre wavelength methanol transitions which are predicted to show weak maser emission towards star formation regions. Sensitive, high spatial, and spectral resolution observations towards four high-mass star formation regions which show emission in a large number of class II methanol maser transitions did not result in any detections. From these observations, we are able to place an upper limit of $\lesssim 1300 \mathrm{~K}$ on the brightness temperature of any emission from the $3_{1} \mathrm{~A}^{+}-3_{1} \mathrm{~A}^{-}, 17_{-2}-18_{-3} \mathrm{E}\left(v_{t}=1\right), 12_{4}-13_{3} \mathrm{~A}^{-}$, $12_{4}-13_{3} \mathrm{~A}^{+}$, and $4_{1} \mathrm{~A}^{+}-4_{1} \mathrm{~A}^{-}$transitions of methanol in these sources on angular scales of 2 arcsec. This upper limit is consistent with current models for class II methanol masers in high-mass star formation regions and better constraints than those provided here will likely require observations with next-generation radio telescopes.
\end{abstract}

Keywords: ISM: molecules - masers - radio lines: ISM - stars: formation

\section{INTRODUCTION}

Methanol is an asymmetric top molecule with hindered internal rotation (Lees 1973), a combination which produces a rich rotational spectrum at centimetre and millimetre wavelengths. Methanol is also a relatively common molecule in some interstellar environments. Two different types of methanol masers are observed in astrophysical environments, known as class I and class II. Class I methanol masers are produced by outflows and other low-velocity shocks, the most common transitions are the 36 and $44 \mathrm{GHz}$ and the emission is distributed over regions of up to 1 pc (e.g. Voronkov et al. 2014). Class II methanol masers are produced by far infrared and submillimetre radiation, they are found close to young high-mass stars and the most common transitions are at 6.7 and $12.2 \mathrm{GHz}$. The empirical division of the maser transitions is consistent with maser modelling which shows that the class I masers arise in regions where collisional processes dominate (e.g. Sobolev et al. 2007; McEwen, Pihlström, \& Sjouwerman 2014), whilst the class II masers are radiatively pumped (e.g. Sobolev \& Deguchi 1994; Cragg, Sobolev, \& Godfrey 2005). Class I methanol masers are predominantly associated with high-mass star formation regions, but have been observed towards low-mass star formation regions (Kalenskii et al. 2010), supernova remnants (Pihlström et al.
2014), and starburst galaxies (Ellingsen et al. 2014; Chen et al. 2015). In contrast, class II methanol masers are only observed associated with high-mass star formation regions (Breen et al. 2013).

To date, more than 30 different class II methanol maser transitions have been observed (see Ellingsen et al. 2012, and references therein). However, compared to the common 6.7 and $12.2 \mathrm{GHz}$ class II methanol transitions, the others are much less common, and where they are observed are weaker than the 6.7 and $12.2 \mathrm{GHz}$ emission (e.g. Caswell et al. 2000; Ellingsen et al. 2004; Cragg et al. 2004; Ellingsen et al. 2011). The output from the methanol maser models includes predictions of the brightness temperature for each of the different transitions. The strong, common class II methanol maser transitions (such as the 6.7 and $12.2 \mathrm{GHz}$ ) are predicted to produce intense maser emission for a wide range of gas temperatures, densities, and methanol column densities (Sobolev, Cragg, \& Godfrey 1997b; Cragg et al. 2005). In contrast, the rarer, weaker methanol transitions are typically inverted over a much narrower range of physical conditions, and the majority of transitions are not expected to be masers. So the presence (or absence) of the rarer, weaker transitions then provides more stringent tests of maser models (Cragg et al. 2004). The relationship between the measured flux density $S$ for emission and the brightness temperature $T_{\mathrm{b}}$ of a source is 
given by the equation

$$
S[\mathrm{Jy}]=5.65 \times 10^{-7} v^{2}[\mathrm{GHz}] \theta^{2}[\operatorname{arcsec}] T_{\mathrm{b}}[\mathrm{K}],
$$

where $v$ is the frequency of the observations and $\theta$ is the angular scale of the emission region. For a source to be detectable at a particular frequency requires either a high brightness temperature, or emission over a large angular scale. Maser sources generally fall into the first of these categories with strong 6.7 and $12.2 \mathrm{GHz}$ methanol masers having brightness temperatures in excess of $10^{10} \mathrm{~K}$ (e.g. Menten et al. 1992). The majority of maser searches are made with singledish telescopes with moderate (around $1 \mathrm{Jy}$ ) sensitivity limits; however, whilst these are efficient for detecting strong masers, they do not place strong upper limits on moderate or low brightness temperature masers $\left(\lesssim 10^{8} \mathrm{~K}\right)$. Sensitive, high resolution observations of some rare class II methanol maser transitions have shown that masers with brightness temperatures of less than $10^{8} \mathrm{~K}$ are likely present in some sources $(\mathrm{Kr}$ ishnan et al. 2013). So rigorous tests of maser models require, sensitive, high resolution observations in order to detect, or limit moderate and low brightness temperature masers. The Australia Telescope Compact Array (ATCA) is an ideal instrument for undertaking such observations and in this paper, we report the results of a search for a number of methanol maser transitions which are predicted to exhibit weak maser emission under some physical conditions.

In order to test and refine maser models and potentially the physical conditions in the observed star formation regions, we selected a number of potential class II methanol maser transitions in the frequency range 5-9 GHz. A search for potential new maser transitions is a compromise between the number of different sources observed and the sensitivity of the observations. For the primary target transitions, we made observations of four high-mass star formation regions which are known to exhibit moderately strong emission from a range of different class II methanol maser transitions. For the secondary target transitions, we made observations of a single source G $345.01+1.79$, as there are more class II methanol maser transitions detected towards that source than any other (Ellingsen et al. 2012).

\section{OBSERVATIONS AND DATA REDUCTION}

We used the ATCA in the 6A configuration (baselines ranging from 337 to $5939 \mathrm{~m}$ ) to undertake sensitive observations of five different methanol $\left(\mathrm{CH}_{3} \mathrm{OH}\right)$ transitions in the $5-9-\mathrm{GHz}$ frequency range. The observations were made over a period of $13 \mathrm{~h}$ on 2000 August 30. The primary target transitions were the $12_{4}-13_{3} \mathrm{~A}^{-}$and $12_{4}-13_{3} \mathrm{~A}^{+}$transitions, which have rest frequencies of 7.682232(50) and 7.830864(50) GHz, respectively (Tsunekawa et al. 1995, the numbers in brackets represent the uncertainty in the least significant figures). These two transitions were observed towards four high-mass star formation regions (G 339.88-1.26, G 345.01+1.79, NGC6334F, and W48), which all exhibit strong class II methanol maser emission in multiple transitions. The pointing centre for the observations of each source and the peak velocity of the class II methanol maser transitions is given in Table 1. For each transition, observations consisted of a series of scans of $5 \mathrm{~min}$ for each target source spread over a range of hour angles. Each scan on a target source was both preceded and followed by a 1 min scan on a nearby phase calibrator. The total observing time for each of the four target maser sources is around $45 \mathrm{~min}$ for each of the primary transitions.

Observations were also undertaken of secondary transitions $3{ }_{1} \mathrm{~A}^{+}-3_{1} \mathrm{~A}^{-}, 17_{-2}-18_{-3} \mathrm{E}\left(v_{t}=1\right)$, and $4_{1} \mathrm{~A}^{+}$ $4_{1} \mathrm{~A}^{-}$which have rest frequencies of 5.0053207(6), 6.181589(153), and 8.341622(4) GHz, respectively (Breckenridge \& Kukolich 1995; Xu \& Lovas 1997). These three transitions were observed only towards the $\mathrm{G} 345.01+1.79$ star formation region with onsource durations of $4.2 \mathrm{~min}$ for each transition. Of the five transitions observed, the only one which has been detected astronomically is the $3{ }_{1} \mathrm{~A}^{+}-3_{1} \mathrm{~A}^{-}$, for which Robinson et al. (1974) report weak, broad emission towards Sgr B2.

The correlator was configured to the FULL_4_1024_POL mode which has 1024 spectral channels across a $4 \mathrm{MHz}$ bandwidth, with all polarisation products recorded. This corresponds to a velocity coverage of approximately $155 \mathrm{~km} \mathrm{~s}^{-1}$ at a frequency of $7.7 \mathrm{GHz}$ and velocity resolution of approximately $0.18 \mathrm{~km} \mathrm{~s}^{-1}$. The data were reduced using MIRIAD, applying the standard data reduction procedures for ATCA observations. The primary flux density calibration was with respect to the standard calibrator PKS B1934-638 using the in-built scale within MIRIAD (Reynolds 1994). The absolute flux density calibration is expected to be accurate to better than 10\%. PKS B1934-638 was also used for bandpass calibration.

\section{RESULTS}

We imaged each of the four sources using the calibrated $4 \mathrm{MHz}$ datasets for the $12_{4}-13_{3} \mathrm{~A}^{-}$and $12_{4}-13_{3} \mathrm{~A}^{+}$transitions. Three of the four sources (all except G 339.88-1.26) have detectable radio continuum emission in both transitions. For these sources, we undertook self-calibration using a model formed from the cleaned images of the radio continuum emission. We found that a single iteration of phase-only self-calibration significantly improved the signal-to-noise ratio in the continuum images and we then used continuum subtraction to extract a spectral line dataset. We imaged a $10 \mathrm{~km} \mathrm{~s}^{-1}$ velocity range covering all the class II methanol maser emission in each source at a velocity resolution of $0.2 \mathrm{~km} \mathrm{~s}^{-1}$ (see Table 1). Inspection of the image cubes for each of the sources did not reveal any emission in either of the two primary target transitions in any of the four source observed. The RMS noise level in $0.2 \mathrm{~km} \mathrm{~s}^{-1}$ spectral channels were in the range $27-37 \mathrm{mJy}^{\text {beam }}{ }^{-1}$ for all sources and are listed in Table 1. Figure 1 shows the continuum images formed from the $12_{4}-13_{3} \mathrm{~A}^{+}$dataset after self-calibration for G 345.01+1.79 and NGC6334F. We do not show the continuum image for W48 because as it is very close to the equator, 
Table 1. Class II methanol maser sources observed in the $12_{4}-13_{3} \mathrm{~A}^{-}$and $12_{4}-13_{3} \mathrm{~A}^{+}$transitions, with the measured RMS noise for each transition, respectively. Distance references ${ }^{a}$ Krishnan et al. (2015); ${ }^{b}$ Green \& McClure-Griffiths (2011); ${ }^{c}$ Wu et al. (2014).

\begin{tabular}{|c|c|c|c|c|c|c|c|}
\hline Source & $\begin{array}{l}\text { Right ascension } \\
\text { (J2000) }\end{array}$ & $\begin{array}{l}\text { Declination } \\
\text { (J2000) }\end{array}$ & $\begin{array}{l}\text { Peak velocity } \\
\left(\mathrm{km} \mathrm{s}^{-1}\right)\end{array}$ & $\begin{array}{l}\text { Velocity range } \\
\left(\mathrm{km} \mathrm{s}^{-1}\right)\end{array}$ & $\begin{array}{l}12_{4}-13_{3} \mathrm{~A}^{-} \\
\text {RMS for transition }\end{array}$ & $\begin{array}{l}12_{4}-13_{3} \mathrm{~A}^{+} \\
\left(\mathrm{mJy} \text { beam }^{-1}\right)\end{array}$ & $\begin{array}{l}\text { Distance } \\
(\mathrm{kpc})\end{array}$ \\
\hline G $339.88-1.26$ & $16: 52: 04.68$ & $-46: 08: 34.4$ & -39.0 & $-44--34$ & 33 & 27 & $2.1^{a}$ \\
\hline G $345.01+1.79$ & $16: 56: 47.58$ & $-40: 14: 25.9$ & -21.0 & $-24--14$ & 35 & 29 & $2.0^{b}$ \\
\hline NGC6334F & $17: 20: 53.45$ & $-35: 47: 00.5$ & -10.0 & $-14--4$ & 36 & 29 & $1.35^{c}$ \\
\hline W48 & 19:01:46.00 & $+01: 12: 29.0$ & +45.0 & $+38-+48$ & 35 & 39 & $3.27^{c}$ \\
\hline
\end{tabular}
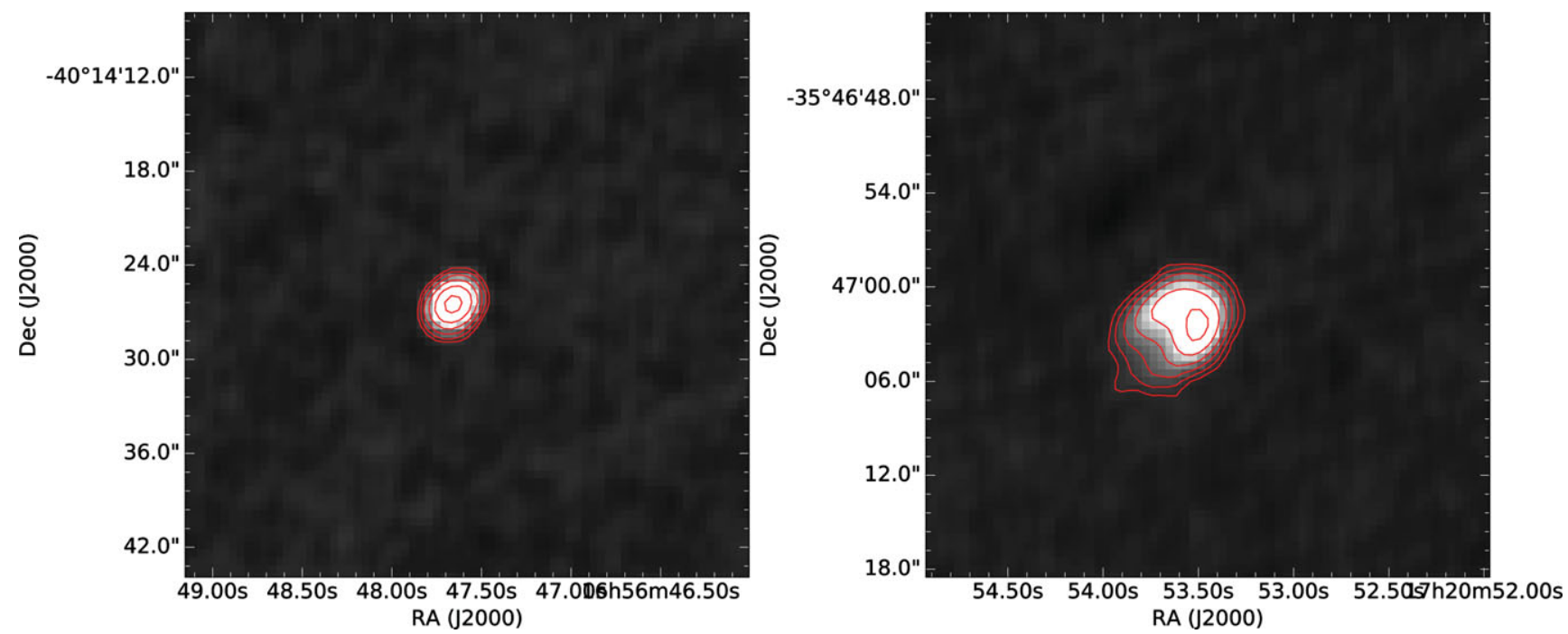

Figure 1. Radio continuum emission at $7.8 \mathrm{GHz}$ for G 345.01+1.79 (left) and NGC6334F (right). For both sources, contour levels are at 2.5, 5, 10, 20, 40 and $80 \%$ of the image peak which is 217 and $776 \mathrm{mJy}^{-1}$ beam ${ }^{-1}$ for G 345.01+1.79 and NGC6334F, respectively. The RMS noise level as are 1.4 and 4.1 mJy beam ${ }^{-1}$ for G $345.01+1.79$ and NGC6334F, respectively. The synthesised beam for these observations was approximately 2.0 arcsec $\times 1.8$ arcsec.

the ATCA as an East-West synthesis array has a very elongated synthesised beam and does not produce good images. Comparison of the images in Figure 1 with previous more sensitive observations of these sources at centimetre wavelengths (e.g. Ellingsen, Shabala, \& Kurtz 2005) shows similar structure and intensity. This demonstrates that despite both of these transitions lying outside the nominal frequency range of the ATCA receiver system (at the time of the observations), the system performed well and the non-detection of the spectral line emission is robust.

The three secondary transitions $\left(3 \mathrm{~A}^{+}-3_{1} \mathrm{~A}^{-}, 17_{-2}-\right.$ $18_{-3} \mathrm{E}\left(v_{t}=1\right)$, and $\left.4_{1} \mathrm{~A}^{+}-4_{1} \mathrm{~A}^{-}\right)$were each observed for 4.2 min towards $G 345.01+1.79$. We have calibrated these data using the same approach as for the primary transitions; however, with a one-dimensional array configuration (such as the ATCA in the 6A array), it is not possible to image data from a single scan. We produced a vector averaged spectrum at the phase centre for each of the transitions and from this we obtained upper limits $(3 \sigma)$ on the intensity of any emission of $0.17,0.15$, and $0.25 \mathrm{Jy}$ for the $3_{1} \mathrm{~A}^{+}-3_{1} \mathrm{~A}^{-}, 17_{-2}-18_{-3} \mathrm{E}$ $\left(v_{t}=1\right)$, and $4_{1} \mathrm{~A}^{+}-4_{1} \mathrm{~A}^{-}$transitions, respectively. The effective field of view for the vector averaged spectrum is the size of the synthesised beam (a few arcseconds), centred on the coordinates given for $\mathrm{G} 345.01+1.79$ (Table 1). This is sufficient to encompass the class II methanol masers in this source which are observed to be confined to a single compact cluster with an angular extent of less than 0.5 arcsec (Krishnan et al. 2013).

\section{DISCUSSION}

Sobolev, Cragg, \& Godfrey (1997a) used the methanol maser pumping model of Sobolev et al. (1997b) to predict which transitions are likely to show maser emission under a range of different physical conditions. In total six different pumping model input parameters were varied and the results for 10 different combinations are listed in Table 1 of Sobolev et al. (1997a). The following model parameters were varied, the gas temperature $\left(T_{\text {kin }}\right)$, the gas density $\left(n_{\mathrm{H}}\right)$, the maser beaming factor $(\epsilon)$, the dilution of continuum emission from a background HII region $\left(W_{\mathrm{H}_{\mathrm{II}}}\right)$, the dust temperature $\left(T_{\mathrm{d}}\right)$, and the methanol specific column density $N_{\mathrm{M}} / \Delta V$. The $12_{4}-$ $13_{3} \mathrm{~A}^{-}$and $12_{4}-13_{3} \mathrm{~A}^{+}$transitions were predicted to exhibit weak maser emission (brightness temperatures of $10^{4}-10^{5} \mathrm{~K}$ ) in 6 of the 10 models, more than any of the other methanol transitions at frequencies $<10 \mathrm{GHz}$ which had not previously been detected. The most recent published class II methanol maser pumping model is that of Cragg et al. (2005), which is 
based on the earlier work of Sobolev et al., but incorporates a number of improvements and refinements, including new collisional data and more energy levels. The Cragg et al. (2005) model also suggests that the $12_{4}-13_{3} \mathrm{~A}^{-}$and $12_{4}-13_{3} \mathrm{~A}^{+}$ transitions are good candidates for weak masers, with six of the eight sets of input physical parameters they trialled yielding masers with brightness temperatures $>10^{3} \mathrm{~K}$, with three of them having brightness temperatures in excess of $10^{4} \mathrm{~K}$.

The four sources targeted for the current search are a subset of the 25 high-mass star formation regions which have been observed to show maser emission from the $107 \mathrm{GHz} 3_{1}-4_{0} \mathrm{~A}^{+}$ transition. Table 5 of Ellingsen et al. (2011) summarises the observed intensity, or measured upper limit for a dozen different class II methanol maser transitions towards these sources and shows that G 339.88-1.26, G 345.01+1.79, NGC6334F, and $\mathrm{W} 48$ have been detected in $6,10,8$, and 5 of these dozen transitions, respectively. Comparing the class II methanol maser transitions observed in our four target sources with the results in Table 2 of Cragg et al. (2005), they best match model 3 , as it is the only model that predicts moderately strong $37.7 \mathrm{GHz}$ methanol masers, which all these sources have. This model has $T_{\text {kin }}=30 \mathrm{~K}, T_{\mathrm{d}}=175 \mathrm{~K}, n_{\mathrm{H}}=10^{7}$ $\mathrm{cm}^{-3}, N_{\mathrm{M}} / \Delta V=3 \times 10^{11} \mathrm{~cm}^{-3} \mathrm{~s}$, and $W_{\mathrm{H}_{\text {II }}}=0.002$. This model predicts brightness temperatures of $\sim 6.3 \times 10^{3} \mathrm{~K}$ for the $12_{4}-13_{3} \mathrm{~A}^{-}$and $12_{4}-13_{3} \mathrm{~A}^{+}$transitions.

Table 1 shows that our most sensitive observations were of the $12_{4}-13_{3} \mathrm{~A}^{+}$transition at $7.83 \mathrm{GHz}$ for which we obtained RMS noise levels in $0.2 \mathrm{~km} \mathrm{~s}^{-1}$ spectral channels of $<30 \mathrm{mJy} \mathrm{beam}^{-1}$ for three of the four sources (we had less time onsource for W48, so the RMS noise is higher). We can be confident that there is no emission stronger than $0.150 \mathrm{Jy}$ beam $^{-1}(5-\sigma)$ in this transition in the observed sources and from equation (1) that places an upper limit of $\sim 1100 \mathrm{~K}$ on the brightness temperature of any emission on angular scales of $2 \operatorname{arcsec}$ (the angular resolution of our observations). This is significantly less than the predicted brightness temperature in the Cragg et al. (2005) model; however, maser emission generally occurs on much smaller angular scales than the 2 arcsec resolution of our synthesised beam. Very long baseline interferometry observations of class II methanol masers (e.g. Menten et al. 1992; Minier, Booth, \& Conway 2002; Harvey-Smith \& Cohen 2006) show that the $6.7 \mathrm{GHz}$ class II methanol masers generally have angular sizes in the range 550 milliarcsec. Rearranging equation (1) for the $12{ }_{4}-13_{3} \mathrm{~A}^{+}$ transition, our $0.15 \mathrm{Jy}$ beam $^{-1}$ upper limit on the flux of the emission from this transition implies $\theta^{2} T_{b}<4330 \mathrm{~K} \operatorname{arcsec}^{2}$. This corresponds to upper limits on the brightness temperature of $1.7 \times 10^{6} \mathrm{~K}$ for emission on scales of 50 milliarcsec or $1.7 \times 10^{8} \mathrm{~K}$ for emission on scales of 5 milliarcsec. These limits are both more than a order of magnitude higher than the predictions of any of the models of either Sobolev et al. (1997a) or Cragg et al. (2005) for this transition.

The observations of the $12{ }_{4}-13_{3} \mathrm{~A}^{-}$transition at $7.62 \mathrm{GHz}$ are approximately $20 \%$ less sensitive than those for the $12_{4}$ $13_{3} \mathrm{~A}^{+}$transition. The lower sensitivity is believed to be due to poorer receiver performance at the frequency of that transi- tion (at the time of our observations). The affect of this is that the limits for the $12{ }_{4}-13_{3} \mathrm{~A}^{-}$transition are $20 \%$ higher; however, this makes no significant difference to the conclusions we can draw.

It should be noted that the analysis undertaken here utilises data with a $0.2 \mathrm{~km} \mathrm{~s}^{-1}$ velocity resolution. This is close to optimal for Galactic maser work, as typical line widths are around $0.5 \mathrm{~km} \mathrm{~s}^{-1}$. Averaging several spectral channels would yield lower RMS noise levels in spectral line cubes; however, the resulting limits would be misleading, as they would not account for the dilution of narrow line emission.

From equation (1), we can estimate the minimum sensitivity for observations of the transitions targeted in this search that would be required to provide a stringent test of methanol maser pumping models. If we take a maximum likely size for the angular scale of the maser emission as 50 milliarcsec, then a maser brightness temperature of $10^{4} \mathrm{~K}$ will result in a flux density of around $1 \mathrm{mJy}$. Robust detection of emission at this level in a $0.2 \mathrm{~km} \mathrm{~s}^{-1}$ spectral channel would require observations about two orders of magnitude more sensitive than those we report here. This level of sensitivity could be achieved with very long (of order days) integrations on sensitive telescopes with large collecting areas (e.g. the GBT, JVLA, or FAST); however, it more realistically lies within the realm of a future SKA survey of line emission in Galactic high-mass star formation regions.

\section{CONCLUSION}

We have searched for emission from a number of centimetre wavelength methanol transitions which are predicted to show weak maser emission towards high-mass star formation regions under some physical conditions. Our observations did not detect emission from any of the transitions in any of the four sources observed with upper limits of approximately $0.15 \mathrm{Jy}$. The observations reported here are approximately an order of magnitude more sensitive and an order of magnitude higher angular resolution than typical single dish searches for maser transitions and so they do represent a significant improvement on many searches for weak, rare class II methanol masers (e.g. Ellingsen et al. 2003; Cragg et al. 2004). Previous observations have demonstrated that more sensitive observations can lead to the detection of weak maser lines, for example, Sobolev et al. (2007) detected weak $23.1 \mathrm{GHz}$ class II methanol masers after earlier unsuccessful searches by Cragg et al. (2004). To significantly improve on the limits placed on the $3_{1} \mathrm{~A}^{+}-3_{1} \mathrm{~A}^{-}, 17_{-2}-18_{-3} \mathrm{E}\left(v_{t}=1\right), 12_{4}-13_{3} \mathrm{~A}^{-}, 12_{4}$ $13_{3} \mathrm{~A}^{+}$, and $4_{1} \mathrm{~A}^{+}-4_{1} \mathrm{~A}^{-}$transitions of methanol by the current observations will require next-generation radio astronomy facilities.

\section{ACKNOWLEDGEMENTS}

The Australia Telescope Compact Array is part of the Australia Telescope which is funded by the Commonwealth of Australia for operation as a National Facility managed by CSIRO. This research 
has made use of NASA's Astrophysics Data System Abstract Service. AC was funded by a University of Tasmania Dean's Summer Research scholarship. AMS has been supported by the Ministry of Education and Science of the Russian Federation within the framework of state work 'Organization of Execution of Scientific Research'. This work was also supported by Government of the Russian Federation Act 211, contract no 02.A03.21.0006.

\section{REFERENCES}

Breckenridge, S. M., \& Kukolich, S. G. 1995, ApJ, 438, 504

Breen, S. L., Ellingsen, S. P., Contreras, Y., Green, J. A., Caswell, J. L., Stevens, J. B., Dawson, J. R., \& Voronkov, M. A. 2013, MNRAS, 435, 524

Caswell, J. L., Yi, J., Booth, R. S., \& Cragg, D. M. 2000, MNRAS, 313, 599

Chen, X., Ellingsen, S. P., Baan, W. A., Qiao, H.-H., Li, J., An, T., \& Breen, S. L. 2015, ApJ, 800, L2

Cragg, D. M., Sobolev, A. M., Caswell, J. L., Ellingsen, S. P., \& Godfrey, P. D. 2004, MNRAS, 351, 1327

Cragg, D. M., Sobolev, A. M., \& Godfrey, P. D. 2005, MNRAS, 360,533

Ellingsen, S. P., Breen, S. L., Sobolev, A. M., Voronkov, M. A., Caswell, J. L., \& Lo, N. 2011, ApJ, 742, 109

Ellingsen, S. P., Chen, X., Qiao, H.-H., Baan, W., An, T., Li, J., \& Breen, S. L. 2014, ApJ, 790, L28

Ellingsen, S. P., Cragg, D. M., Minier, V., Muller, E., \& Godfrey, P. D. 2003, MNRAS, 344, 73

Ellingsen, S. P., Cragg, D. M., Lovell, J. E. J., Sobolev, A. M., Ramsdale, P. D., \& Godfrey, P. D. 2004, MNRAS, 354, 401

Ellingsen, S. P., Shabala, S. S., \& Kurtz, S. E. 2005, MNRAS, 357 , 1003

Ellingsen, S. P., Sobolev, A. M., Cragg, D. M., \& Godfrey, P. D. 2012, ApJ, 759, L5

Green, J. A., \& McClure-Griffiths, N. M. 2011, MNRAS, 417, 2500

Harvey-Smith, L., \& Cohen, R. J. 2006, MNRAS, 371, 1550
Kalenskii, S. V., Johansson, L. E. B., Bergman, P., Kurtz, S., Hofner, P., Walmsley, C. M., \& Slysh, V. I. 2010, MNRAS, 405,613

Krishnan, V., Ellingsen, S. P., Voronkov, M. A., \& Breen, S. L. 2013, MNRAS, 433, 3346

Krishnan, V., et al. 2015, ApJ, 805, 129

Lees, R. M. 1973, ApJ, 184, 763

McEwen, B. C., Pihlström, Y. M., \& Sjouwerman, L. O. 2014, ApJ, 793, 133

Menten, K. M., Reid, M. J., Pratap, P., Moran, J. M., \& Wilson, T. L. 1992, ApJ, 401, L39

Minier, V., Booth, R. S., \& Conway, J. E. 2002, A\&A, 383, 614

Pihlström, Y. M., Sjouwerman, L. O., Frail, D. A., Claussen, M. J., Mesler, R. A., \& McEwen, B. C. 2014, AJ, 147, 73

Reynolds, J. 1994, Technical report, Technical Report 39.3/040, A Revised Flux Scale Technical Report 39.3/040, A Revised FLux Scale for the AT Compact Array. ATNF

Robinson, B. J., Brooks, J. W., Godfrey, P. D., \& Brown, R. D. 1974, AuJPA, 27, 865

Sobolev, A. M., Cragg, D. M., \& Godfrey, P. D. 1997a, MNRAS, 288, L39

Sobolev, A. M., Cragg, D. M., \& Godfrey, P. D. 1997b, A\&A, 324, 211

Sobolev, A. M., \& Deguchi, S. 1994, A\&A, 291, 569

Sobolev, A. M., et al. 2007, in IAU Symp., Vol. 242, Astrophysical Masers and their Environments, eds. J. M. Chapman, \& W. A. Baan (Cambridge: Cambridge University Press), 81 (arXiv 0706.3117), doi:10.1017/S1743921307012616

Tsunekawa, S., Ukai, T., Toyama, A., \& Takagi, K. 1995, Technical report, Report for the Grant-in-aid for Scientific Research on Priority Areas (Interstellar Matter, 1991-1994) of hte Ministry of Education, Science and Culture, Japan. Toyama University

Voronkov, M. A., Caswell, J. L., Ellingsen, S. P., Green, J. A., \& Breen, S. L. 2014, MNRAS, 439, 2584

Wu, Y. W., et al. 2014, A\&A, 566, A17

Xu, L.-H., \& Lovas, F. J. 1997, JPCRD, 26, 17 